\title{
Determination of Tissue Optical Properties by Steady-State Spatial Frequency-Domain Reflectometry
}

\author{
N. Dögnitz and G. Wagnières \\ Institute of Environmental Engineering, Swiss Federal Institute of Technology (EPFL), Lausanne, Switzerland
}

\begin{abstract}
A new non-invasive method to measure the optical properties of biological tissue is described. This method consists of illuminating the investigated sample with light which is spatially periodically modulated in intensity. The spatial modulation of the backscattered light and the diffuse reflectivity of the sample, both detected with an imaging technique, are used to deduce the absorption and reduced scattering coefficient from a table generated by Monte Carlo simulations. This principle has three major advantages: Firstly, it permits the immediate acquisition of the average values of the optical coefficients over a relatively large area (typ. $20 \mathrm{~mm}$ in diameter), thus avoiding the perturbations generated by small tissue heterogeneities; It also provides good flexibility for measuring the optical coefficients at various wavelengths and it does not require the use of a detector with a large dynamic range. The method was first validated on phantoms with known optical properties. Finally, we measured the optical properties of human skin at $400 \mathrm{~nm}, 500 \mathrm{~nm}, 633 \mathrm{~nm}$ and $700 \mathrm{~nm}$ in vivo.
\end{abstract}

Keywords: Absorption coefficient; Optical properties; Reduced scattering coefficient; Skin; Spatial frequency-domain reflectometry; Tissue optics

\section{INTRODUCTION}

A knowledge of light distribution in living tissue is useful in predicting, analysing and understanding the photobiological, photophysical, and photochemical processes which occur in such a medium. Numerous models of light distribution in homogeneous turbid media have been described in recent years. Most of them are based on the transport equation and the diffusion approximation [1-3]. The distribution of red and near infrared light in tissue can be obtained with analytical solutions of the diffusion equation for simple geometrical conditions or with numerical techniques such as Monte Carlo simulations [4,5]. These solutions are used, for example, to optimise the photodynamic therapy of cancer [6] or to model and optimise the characterisation of tissues using light-induced fluorescence spectroscopy $[7,8]$.

A turbid medium can be characterised by four optical parameters: the absorption $\mu_{\mathrm{a}}$

Correspondence to: Georges Wagnières, EPFL-DGR-LPAS, CH-H5, CH-1015 Lausanne, Switzerland. (mm ${ }^{-1}$ ) and the scattering $\mu_{\mathrm{s}}\left(\mathrm{mm}^{-1}\right)$ coefficients defining the probability $p$ for a photon to be absorbed $\left(p=1-\exp \left(-\mu_{\mathrm{a}} z\right)\right)$ and scattered $\left(p=1-\exp \left(-\mu_{\mathrm{s}} z\right)\right)$ respectively in the pathlength $z$, the phase function describing the probability density for a photon to be scattered in a certain direction, and the refractive index $n_{\text {tissue }}$. In the case of an anisotropic phase function, as found in biological tissue, the Henyey-Greenstein [9] and Rayleigh-Gans $[10,11]$ functions are often used. The scattering is described by the average cosine $g$ of the scattering angle. Typical values of $g$ range between 0.8 and 0.95 [12] for soft mammalian tissues. The reduced scattering coefficient $\mu_{\mathrm{s}}{ }^{\prime}=\mu_{\mathrm{s}}(1-g)$ can be used to describe the scattering, if two kinds of scattering media give the same light distribution, which have identical $\mu_{\mathrm{a}}$ but different anisotropy parameter $g$ and scattering coefficient $\mu_{\mathrm{s}}$. This is generally accurate if the medium is very diffusing, i.e. $\mu_{\mathrm{s}}{ }^{\prime}>>\mu_{\mathrm{a}}$ and if the light distribution is studied where it is not close to boundaries and the illumination point $[13,14]$. Often it is appropriate to describe the propagation of light by the diffusion model parameter $\mu_{\text {eff }}\left(\mathrm{mm}^{-1}\right)$, the 
effective attenuation coefficient. It is defined by the relation: $\mu_{\mathrm{eff}}=\left[3 \mu_{\mathrm{a}}\left(\mu_{\mathrm{a}}+\mu_{\mathrm{s}}{ }^{\prime}\right)\right]^{1 / 2}$. Its inverse is the effective penetration depth of light.

Several groups have proposed in vivo and ex vivo methods to determine the optical parameters of tissues. Ex vivo measurements are obviously more simple to perform than the in vivo ones, however the change of blood contents, tissue architecture, oxygenation, etc. between the in vivo and ex vivo conditions as well as the handling of the tissue sample significantly change the optical coefficients after excision [15]. Several methods were, therefore, investigated in order to obtain the tissue optical coefficients in a non-invasive way. Among these were the following: pulsed photothermal radiometry [16], time-resolved reflectometry [17], frequency-domain reflectometry [18], and relative $[19,20]$ and absolute [21] steady-state spatially resolved diffuse reflectometry. One reported main drawback of these 'local' techniques is related to the large fluctuations generated by tissue inhomogeneities located at the illumination and/or detection positions [22].

The method presented below means that such artefacts can be avoided by probing a relatively large area in vivo (typ. $10-20 \mathrm{~mm}$ in diameter) while preserving the non-invasive aspects and the possible endoscopic use. The measurement method, although based on Monte Carlo simulations, gives the necessary information for a medical application in a time frame compatible to the clinical schedule. Further advantages include good flexibility to measure the optical coefficients at various wavelengths and the fact that the method does not require the use of a detector (CCD) with as large a dynamic range as the standard spatially resolved reflectometry.

The validation of the steady-state spatial frequency-domain reflectometry (SFDR) method, performed on optical phantoms with known optical properties, is presented below. Finally, as illustration of the capabilities of this method, we report on measurements of the optical properties of the human skin in vivo at $400 \mathrm{~nm}, 500 \mathrm{~nm}, 633 \mathrm{~nm}$ and $700 \mathrm{~nm}$.

\section{MATERIALS AND METHODS}

\section{Method}

The principle of the SFDR method consisting of an instrumental and a simulation part is

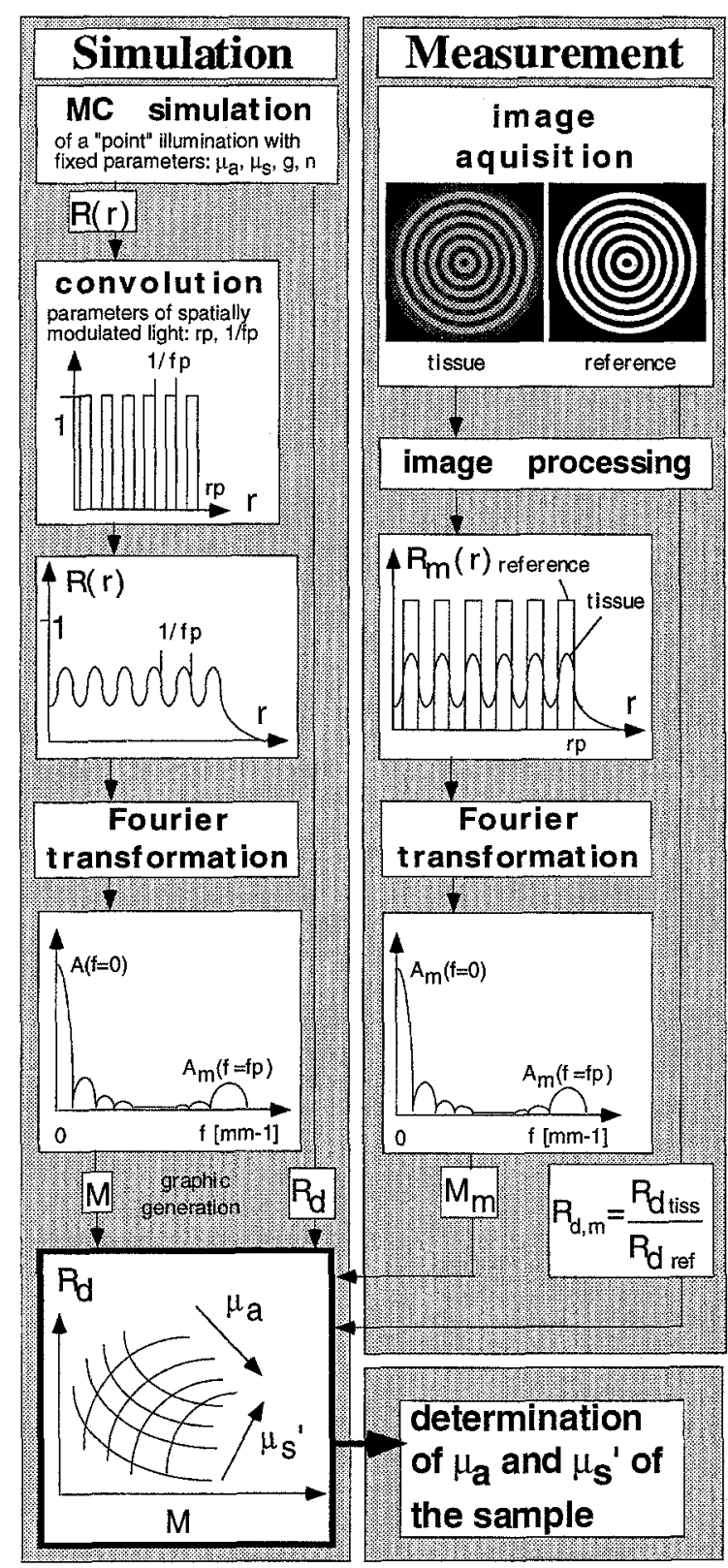

Fig. 1. Principle of the SFDR method.

presented in Fig. 1. In the simulation part, a table is generated on the basis of a Monte Carlo simulation by Wang and Jacques [23] from which the absorption coefficient $\mu_{\mathrm{a}}$ and the reduced scattering coefficient $\mu_{\mathrm{s}}{ }^{\prime}$ can be determined graphically. The table relates the optical coefficients to the diffuse reflectivity of the sample and to the modulation of the backscattered light which can both be measured. In the Monte Carlo simulation, a semi-infinite, isotropic and homogeneous tissue is characterised by its absorption coefficient $\mu_{\mathrm{a}}$, the scattering coefficient $\mu_{\mathrm{s}}$, the anisotropy factor $g$, the refractive index $n$ and the layer 
thickness. The diffuse reflectivity $R_{\mathrm{d}}$ of the simulated tissue and the spatial resolved reflectance $R(r)$ are calculated for an infinitely narrow light beam incident perpendicularly to the air-tissue interface (radial symmetric geometry). All photons leaving the simulated tissue with different angles are taken into account to calculate the diffuse reflectivity and spatial resolved reflectance.

Then, a convolution is performed to yield the spatially resolved reflectance for incident light which is spatially modulated in intensity. The intensity profile of the incident light along the radius is a square wave profile. In other words, the illumination pattern consists of dark and bright concentric rings of equal width, beginning with a dark area in the centre. The pattern is characterised by its spatial frequency $f_{\mathrm{p}}\left(\mathrm{mm}^{-1}\right)$, the number of bright rings $N$ and the pattern radius $r_{\mathrm{p}}=(N-0.25) / f_{\mathrm{p}}$. After the convolution, the spatially resolved reflectance $R(r)$ is Fourier transformed and the amplitudes $A(f=0)$ and $A\left(f=f_{\mathrm{p}}\right)$ are obtained. The modulation of the backscattered light is calculated as $M=2 A\left(f_{\mathrm{p}}\right) / A(0)$. It is noteworthy that for a sample without lateral light propagation in the material, i.e. $\mu_{\mathrm{s}}{ }^{\prime}=\infty$ and $\mu_{\mathrm{a}}>0$, the modulation is $M=4 / \pi$. The Monte Carlo simulation and convolution to calculate the diffuse reflectivity and the modulation of the backscattered light are repeated for various values of the absorption and reduced scattering coefficients, but for the same illumination pattern in order to generate the conversion table.

To determine the optical properties of a tissue sample, the sample investigated and a $100 \%$ reflecting reference material are illuminated with light modulated in intensity corresponding to the illumination pattern used for the convolution. The backscattered light from the tissue sample and from the reference material is detected with an imaging CCD camera and then analysed to determine the spatial modulation $M_{\mathrm{m}}$ of the backscattered light and the diffuse reflectivity $R_{\mathrm{d}, \mathrm{m}}$ of the sample (the index $m$ indicates measured values). The pixel intensities of the tissue and the reference images are integrated to obtain the diffuse reflectivity $R_{\mathrm{d}, \mathrm{m}}$ of the tissue sample by their ratio: $R_{\mathrm{d}, \mathrm{m}}=R_{\mathrm{d}, \mathrm{tiss}} / R_{\mathrm{d} \text {,ref. }}$. After determining the pattern centre point, the spatially resolved reflectance $R_{\mathrm{m}}(r)$ is calculated by averaging the pixel intensity values of the tissue image over all angles between 0 and $2 \pi$. The resulting light intensity profile is Fourier transformed and the amplitudes $A_{\mathrm{m}}(f=0)$ and
$A_{\mathrm{m}}\left(f=f_{\mathrm{p}}\right)$ are obtained. The modulation is given by $M_{\mathrm{m}}=\left[2 A_{\mathrm{m}}\left(f_{\mathrm{p}}\right) / A_{\mathrm{m}}(0)\right] / M T F\left(f_{\mathrm{p}}\right)$, where $\operatorname{MTF}\left(f_{\mathrm{p}}\right)$ is the modulation transfer function of the imaging system at the spatial frequency $f_{\mathrm{p}}$. It was determined by taking an image of a backilluminated $5 \mu \mathrm{m}$ pin hole placed in the same plane as the sample. The $5 \mu \mathrm{m}$ light spot was assumed to be an infinitesimal point. The image of the point is the spatial impulse response of the imaging system and the $M T F$ is given by the normalised Fourier transform of the spatial impulse response [24]. The measurement of the $M T F$ was repeated when a measurement parameter, for example the distance between tissue surface and CCD camera or the wavelength was changed.

With the measured diffuse reflectivity of the tissue sample and the modulation of the backscattered light the absorption coefficient $\mu_{\mathrm{a}}$ and the reduced scattering coefficient $\mu_{\mathrm{s}}^{\prime}$ can be deduced from the table generated by Monte Carlo simulations and convolutions.

\section{Sensitivity of the Method}

Different illumination patterns with a various number of bright rings, pattern radii and spatial pattern frequencies can be used to measure the optical properties in a range corresponding to that of soft biological tissues.

First, the influence of the pattern radius on the modulation was investigated numerically. Monte Carlo simulations were calculated for $\mu_{\mathrm{a}}$ between 0.025 and $0.25 \mathrm{~mm}^{-1}$ and $\mu_{\mathrm{s}}^{\prime}$ between 0.5 and $2.5 \mathrm{~mm}^{-1} \quad(g=0.95$, $n_{\text {tissue }}=1.37$ ). Convolutions were performed for the following conditions: pattern radius between 1.5 and $15.5 \mathrm{~mm} ; N$ between 1 and 8 ; $f_{\mathrm{p}}=0.5 \mathrm{~mm}^{-1}$.

For a given pattern frequency and for values of $r_{\mathrm{p}}>>1 / \mu_{\mathrm{eff}}$, the modulation does not depend on the pattern radius. Effectively, both amplitudes $A(f=0)$ and $A\left(f=f_{\mathrm{p}}\right)$ of the Fourier transformation increase linearly with the pattern radius, resulting in a constant modulation. If the condition $r_{\mathrm{p}}>>1 / \mu_{\mathrm{eff}}$ is not satisfied, the modulation decreases with increasing pattern radius. This effect was seen up to $r_{\mathrm{p}}=11.5 \mathrm{~mm} \quad(N=6)$ for the conditions as described above. Therefore, the influence of the pattern spatial frequency on the sensitivity of the SFDR method was always investigated for large pattern radii between 31.5 and $37.5 \mathrm{~mm}$ with $r_{\mathrm{p}}>>1 / \mu_{\mathrm{eff}}$. 

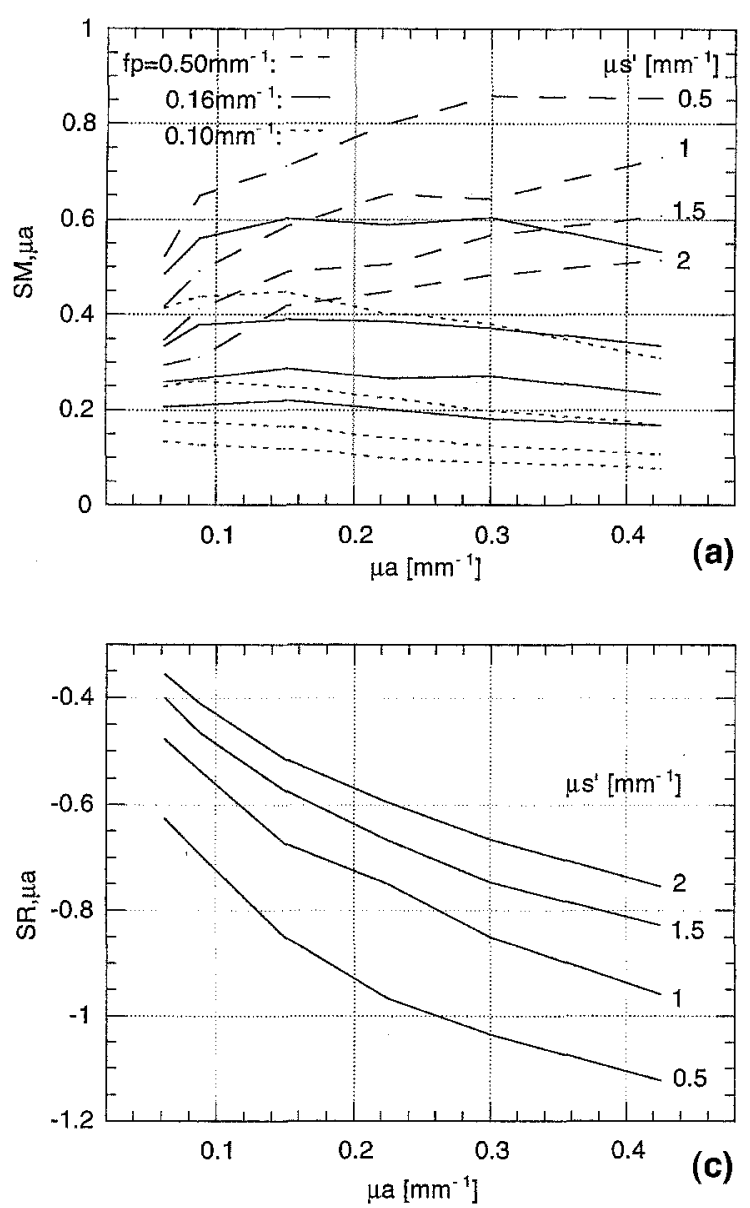

Fig. 2. (a) Sensitivity $S_{M, \mu_{a}}$; (b) sensitivity $S_{M, \mu_{s} ;} ;$ (c) sensitivity $S_{R_{d^{\prime}, \mu_{a}}}$ simulation parameters see text.

To find the optical pattern frequency, the influence on the sensitivity of the SFDR method was investigated numerically. Monte Carlo simulations were calculated for several combinations of the optical properties between $\mu_{\mathrm{a}}=0.05-0.5 \mathrm{~mm}^{-1}$ and $\mu_{\mathrm{s}}{ }^{\prime}=0.5-2 \mathrm{~mm}^{-1}$ $\left(g=0.95, n_{\text {tissue }}=1.37\right)$. Convolutions for three different pattern frequencies $f_{\mathrm{p}}=0.5,0.16$ and $0.1 \mathrm{~mm}^{-1}$ were calculated for all Monte Carlo simulations in order to generate three conversion tables to deduce the optical properties. The typical computation time to generate such a table from Monte Carlo simulations and convolutions is approximately one week with a Sun Sparc station 10/41.

The following analytical development was used to find the optical pattern frequency. The relative variations $E_{M}$ and $E_{R_{d}}$ of the modulation and diffuse reflectivity depend on the relative variations $E_{\mu_{\mathrm{a}}}$ and $E_{\mu_{\mathrm{s}^{\prime}}}$ of the optical parameters:

$$
\begin{aligned}
& E_{M}=S_{M, \mu_{\mathrm{a}}} E_{\mu_{\mathrm{a}}}+S_{M, \mu_{\mathrm{s}}}{ }^{\prime} E_{\mu_{\mathrm{s}^{\prime}}} \\
& E_{R_{\mathrm{d}}}=S_{R_{\mathrm{d}, \mu_{\mathrm{a}}}} E_{\mu_{\mathrm{a}}}+S_{R_{\mathrm{d}, \mu_{\mathrm{s}}}{ }^{\prime} E_{\mu_{\mathrm{s}^{\prime}}}}
\end{aligned}
$$
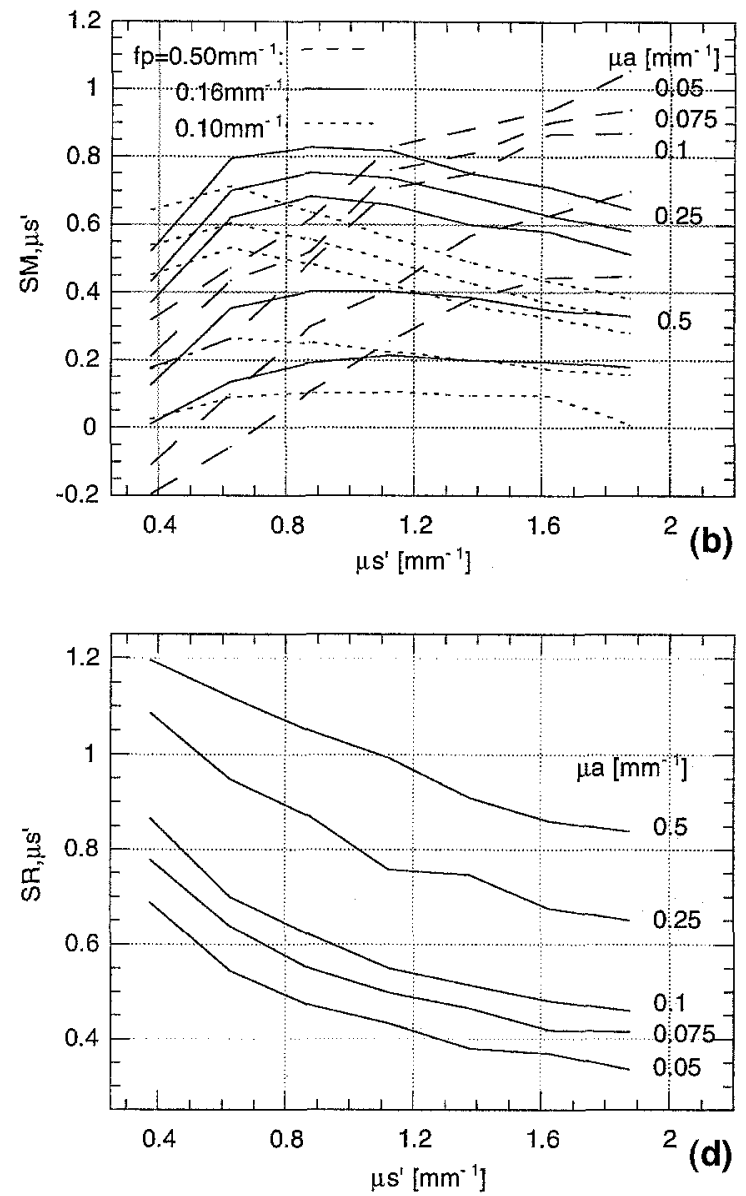
; (d) sensitivity $S_{R_{\mathrm{d}, u_{\mathrm{s}^{\prime}}}} f_{\mathrm{p}}=0.5,0.16$ and $0.1 \mathrm{~mm}^{-1}$. For Thereby, the sensitivities of the modulation $S_{M}$ and of diffuse reflectivity $S_{R_{\tilde{d}}}$ to relative variations of $\mu_{\mathrm{a}}$ and $\mu_{\mathrm{s}}{ }^{\prime}$ are defined as:

$$
\begin{aligned}
S_{M, \mu_{\mathrm{a}}} & =(\Delta M / M)\left(\Delta \mu_{\mathrm{a}} / \mu_{\mathrm{a}}\right)^{-1} \mid \mu_{\mathrm{s}}{ }^{\prime}=\text { const. } \\
S_{M, \mu_{\mathrm{s}}}{ }^{\prime} & =(\Delta M / M)\left(\Delta \mu_{\mathrm{s}}{ }^{\prime} / \mu_{\mathrm{s}}{ }^{\prime}\right)^{-1} \mid \mu_{\mathrm{a}}=\text { const. } \\
S_{R_{\mathrm{d}}, \mu_{\mathrm{a}}} & =\left(\Delta R_{\mathrm{d}} / R_{\mathrm{d}}\right)\left(\Delta \mu_{\mathrm{a}} / \mu_{\mathrm{a}}\right)^{-1} \mid \mu_{\mathrm{s}}{ }^{\prime}=\text { const. } \\
S_{R_{\mathrm{d}}, \mu_{\mathrm{s}}}{ }^{\prime} & =\left(\Delta R_{\mathrm{d}} / R_{\mathrm{d}}\right)\left(\Delta \mu_{\mathrm{s}}{ }^{\prime} / \mu_{\mathrm{s}}{ }^{\prime}\right)^{-1} \mid \mu_{\mathrm{a}}=\text { const. }
\end{aligned}
$$

They can be determined from the conversion tables calculated by the Monte Carlo simulations and convolutions. The sensitivities shown in Fig. 2(a-d) have been determined for $\mu_{\mathrm{a}}=0.05-0.5 \mathrm{~mm}^{-1}$ and $\mu_{\mathrm{s}}{ }^{\prime}=0.5-2 \mathrm{~mm}^{-1}$ $\left(g=0.95, n_{\text {tissue }}=1.37\right)$ and for three different pattern frequencies $f_{\mathrm{p}}=0.5 \mathrm{~mm}^{-1} \quad(N=16)$, $0.16 \mathrm{~mm}^{-1}(N=6), 0.1 \mathrm{~mm}^{-1}(N=4)$. As can be seen in Fig. 2a, $S_{M, \mu_{2}}$ increases for increasing spatial frequencies which is also the case for $S_{M, \mu_{\mathrm{s}}}{ }^{\prime}$ (Fig. 2b), if $\mu_{\mathrm{s}}^{\prime}$ is larger than $1.1 \mathrm{~mm}^{-1}$. The change of sensitivity can be explained as follows. The modulation of the backscattered light reaches its maximum, resulting in low 
Table 1. Maximal relative errors $E_{\mu_{\mathrm{a}}}$ and $E_{\mu_{\mathrm{s}}}{ }^{\prime}$ for $\mu_{\mathrm{a}}=0.0625 \mathrm{~mm}^{-1} \quad \mu_{\mathrm{s}}{ }^{\prime}=1.875 \mathrm{~mm}^{-1}, \quad E_{\mathrm{M}}= \pm 0.05$, $E_{\mathrm{rd}}= \pm 0.02$ and three different pattern spatial frequencies

Relative errors

$E_{\mu_{\mathrm{a}}}$ and $E_{\mu_{\mathrm{s}}}{ }^{\prime}$

$f_{\mathrm{p}}=0.5 \mathrm{~mm}^{-1}$

$f_{\mathrm{p}}=0.166 \mathrm{~mm}^{-1}$

$f_{\mathrm{p}}=0.1 \mathrm{~mm}^{-1}$

$$
\begin{aligned}
& E_{\mu_{\mathrm{a}}}=0.08, E_{\mu_{\mathrm{s}}^{\prime}}=0.05 \\
& E_{\mu_{\mathrm{a}}}=0.10, E_{\mu_{\mathrm{s}}^{\prime}}=0.07 \\
& E_{\mu_{\mathrm{a}}}=0.14, E_{\mu_{\mathrm{s}}}=0.11
\end{aligned}
$$

sensitivity if the effective penetration depth is much smaller than the inverse of the pattern frequency, i.e. if only a minor proportion of the light penetrates into the non-illuminated pattern areas. On the other hand, the modulation reaches its minimum, also resulting in a low sensitivity, if the effective penetration depth is much larger than the inverse of the pattern frequency, i.e. if the light penetrates a long way through the non-illuminated pattern areas.

The sensitivities $S_{R, \mu_{\mathrm{a}}}$ and $S_{R, \mu_{\mathrm{s}}}$ ', which are shown in Fig. 2c and $\mathrm{d}$ depend only on the optical properties and not on the spatial pattern frequency.

The relative error of the optical parameters due to the relative measurement error of $R_{\mathrm{d}, \mathrm{m}}$ and $M_{\mathrm{m}}$ can be obtained by resolving equations (1a) and (1b) for $E_{\mu_{\mathrm{a}}}$ and $E_{\mu_{\mathrm{s}}}$ :

$$
\begin{aligned}
& E_{\mu_{s^{\prime}}}=\left(S_{M, \mu_{\mathrm{s}}} / S_{M, \mu_{\mathrm{a}}}\right. \\
& \left.\stackrel{-S_{s^{\prime}}}{-} S_{R_{\mathrm{d}}, \mu_{\mathrm{s}}}{ }^{\prime \mathrm{s}} / S_{R_{\mathrm{d}}, \mu_{\mathrm{a}}}\right)^{-1}\left(E_{M} / S_{M, \mu_{\mathrm{a}}}-E_{R_{\mathrm{d}}} / S_{R_{\mathrm{d}}, \mu_{\mathrm{a}}}\right) \\
& E_{\mu_{\mathrm{a}}}=\left(S_{M, \mu_{\mathrm{a}}} / S_{M, \mu_{\mathrm{s}}}\right. \\
& -S_{R_{\mathrm{d}}, \mu_{\mathrm{a}}} / S_{R_{\mathrm{d}}, \mu_{\mathrm{s}}}{ }^{\prime}-1\left(E_{M} / S_{M, \mu_{\mathrm{s}}}{ }^{\prime}-E_{R_{\mathrm{d}}} / S_{R_{\mathrm{d}}, \mu_{\mathrm{s}}}{ }^{\prime}\right)
\end{aligned}
$$

$E_{\mu_{\mathrm{a}}}$ and $E_{\mu_{\mathrm{s}^{\prime}}}$ have been calculated for $\mu_{\mathrm{a}}$ between 0.0625 and $0.425 \mathrm{~mm}^{-1}$, for $\mu_{\mathrm{s}}{ }^{\prime}$ between 0.825 and $1.875 \mathrm{~mm}^{-1}$, and for $f_{\mathrm{p}}=0.5$, 0.166 and $0.1 \mathrm{~mm}^{-1}$ from equations $(3 \mathrm{a}, \mathrm{b})$ with the sensitivities shown in Fig. 2(a-d) and with the relative measurement errors $E_{R_{\mathrm{\alpha}}}= \pm 0.02$, $E_{M}= \pm 0.05$ which were assumed as typical measurement errors of $R_{\mathrm{d}, \mathrm{m}}$ and $M_{\mathrm{m}} \cdot E_{\mu_{\mathrm{a}}}$ and $E_{\mu_{s^{\prime}}}$ are always smaller than 0.1 for this range of the optical properties and the spatial frequency $f_{\mathrm{p}}=0.5 \mathrm{~mm}^{-1}$, in contrast to $f_{\mathrm{p}}=0.16 \mathrm{~mm}^{-1}$ and $f_{\mathrm{p}}=0.1 \mathrm{~mm}^{-1}$. An example of the relative errors of $E_{\mu_{\mathrm{a}}}$ and $E_{\mu_{\mathrm{s}^{\prime}}}$ for $\mu_{\mathrm{a}}=0.0625 \mathrm{~mm}^{-1}$ and $\mu_{\mathrm{s}}{ }^{\prime}=1.875 \mathrm{~mm}^{-1}$ which correspond to the range of optical properties of human skin at $633 \mathrm{~nm} \mathrm{[25]} \mathrm{and} \mathrm{to} \mathrm{the}$ optical phantoms presented below is given in Table 1. The illumination pattern with $f_{\mathrm{p}}=0.5 \mathrm{~mm}^{-1}$ results in smaller errors of the

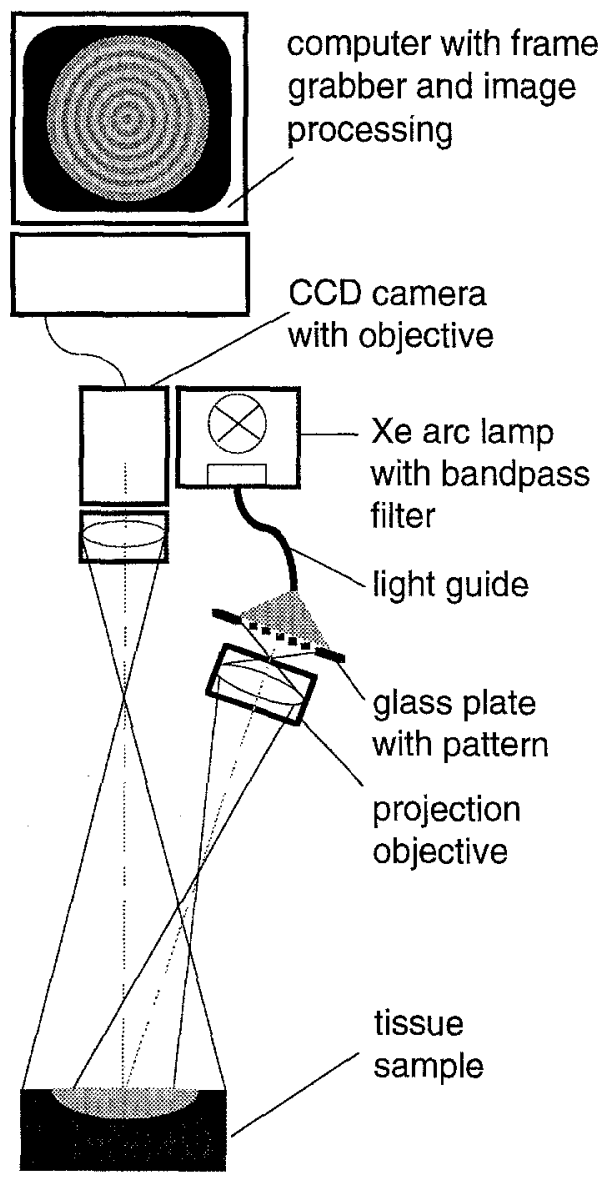

Fig. 3. Experimental set-up to measure $\mu_{\mathrm{a}}$ and $\mu_{\mathrm{s}}^{\prime}$ of a tissue sample with the SFDR method.

optical properties. It was used for all following measurements.

\section{Instrumentation}

The experimental set-up to measure $R_{\mathrm{d}, \mathrm{m}}$ and $M_{\mathrm{m}}$ is presented in Fig. 3. Light from a xenon arc lamp (Lambda DG4, Sutter Instrument Company, Novato, CA, USA) filtered with interference bandpass filters $(\lambda=400,500$, $700 \mathrm{~nm}$, diameter $=25 \mathrm{~mm}, \quad F W H M=40 \mathrm{~nm}$, Andover, Salem, NH, USA; $\lambda=632 \mathrm{~nm}$, diameter $=25 \mathrm{~mm}, \quad F W H M=20 \mathrm{~nm}, \quad$ Edmund Scientific, Barrington, NJ, USA) was coupled into a liquid light guide (Fluid-Lichtkabel, Karl Storz GmbH, Tuttlingen, Germany) to illuminate homogeneously a glass plate with the pattern (chrom mask, pattern: diameter $=1.55 \mathrm{~mm}, N=8$, Photonics MZD, Dresden, Germany). The image of the pattern is magnified and focused on the surface of the sample with a projection objective (AF Nikkor, $f=50 \mathrm{~mm}, 1: 1.8$, Nikon). The spatial 
frequency $f_{\mathrm{p}}=0.5 \mathrm{~mm}^{-1}$ on the sample surface corresponded to the frequency used for the generation of the graphic by Monte Carlo simulations. The sample was illuminated at an angle of $\alpha=7^{\circ}$ to avoid the detection of specular reflections. The power of light generating the pattern on the sample is about $1 \mathrm{~mW}$. The backscattered light was detected by a b/w 16 bit slow scan cooled CCD camera (ST-6, Santa Barbara Instrument Group, Santa Barbara, USA), and a focused image of the sample surface was taken with a telephoto-lens (AF Nikkor, $f=75-210 \mathrm{~mm}, 1: 4-5.6$, Nikon). The image was digitised by the 16 bit A/D converter associated with the camera. The image acquisition time was less than $0.5 \mathrm{~s}$. The acceptance angle of detection was $1.25^{\circ}$. Two pictures were taken under the same conditions (camera integrating time, etc.), one of the tissue sample and the second of a 100\% diffuse reflecting reference sample (white reflectance coating, Eastman Kodak Company, Rochester, NY, USA). Image processing was performed using commercially available software (MATLAB, Mathworks Inc., Natick, MA, USA) with a Sun Sparc station 10/41. The background was subtracted from the two images.

The graphic to determine the optical coefficients from a measured set of $R_{\mathrm{d}, \mathrm{m}}$ and $M_{\mathrm{m}}$ was generated using the following parameters: $N=8, r_{\mathrm{p}}=15.5 \mathrm{~mm}, f_{\mathrm{p}}=0.5 \mathrm{~mm}^{-1}, n_{\text {tissue }}=1.37$, $10^{6}$ photons, $g=0.95, \quad \mu_{\mathrm{a}}=0.01-1 \mathrm{~mm}^{-1}$, $\mu_{\mathrm{s}}{ }^{\prime}=0.25-5 \mathrm{~mm}^{-1}$, homogeneous semi-infinite sample. The optical coefficients located between the calculated grid points of the graphic were interpolated linearly.

\section{Optical Phantoms and Tissue Samples}

The SFDR method was first validated on optical phantoms with known optical properties. The liquid phantoms were made of deionised water loaded with a controlled amount of Lipovenös 20\% (Fresenius AG, Stans, Switzerland) for scattering and China ink (Rotring, Hamburg, Germany) for absorption. The absorption coefficient of China ink dissolved in deionised water was deduced from the absorbance $A_{\mathrm{b}}$ measured with a spectrophotometer (UVkon, Kontron Instruments, USA) with the expression $\mu_{\mathrm{a}}=A_{\mathrm{b}} /(d \log (\mathrm{e}))$, where $d$ is the thickness of the cuvette. A large quantity of this solution was prepared in order to use the same solution for all phantoms. The reduced scattering coefficient of Lipovenös in water was calculated on the basis of measurements published by van Staveren et al. [26]. A solution of $15 \mathrm{~g}$ Lipovenös and $405 \mathrm{~g} \mathrm{H}_{2} \mathrm{O}$ gives a phantom with $\mu_{\mathrm{s}}{ }^{\prime}=0.93 \mathrm{~mm}^{-1}(633 \mathrm{~nm})$, which is large enough to be assumed as semiinfinite (diameter $=100 \mathrm{~mm}, 50 \mathrm{~mm}$ thick).

Five of the phantoms had identical values of $\mu_{\mathrm{s}}{ }^{\prime}=0.93 \mathrm{~mm}^{-1}$ with different values of $\mu_{\mathrm{a}}$ between $0.012 \mathrm{~mm}^{-1}$ and $0.054 \mathrm{~mm}^{-1}$. Eight of the phantoms had identical values of $\mu_{\mathrm{a}}=0.054 \mathrm{~mm}^{-1}$ with different values of $\mu_{\mathrm{s}}{ }^{\prime}$ between $0.93 \mathrm{~mm}^{-1}$ and $1.8 \mathrm{~mm}^{-1}$. It was assumed that increasing the absorption coefficient by adding a small additional amount of ink solution to the phantom would not decrease the reduced scattering coefficient by dilution. The same was assumed for the influence of an additional amount of Lipovenös on the absorption coefficient. Calculations showed that increasing the reduced scattering coefficient of the described phantoms from $\mu_{\mathrm{s}}{ }^{\prime}=0.93 \mathrm{~mm}^{-1}$ to $1.8 \mathrm{~mm}^{-1}$ by adding Lipovenös results only in a decrease of the absorption coefficient by $3 \%$.

A second indirect method, described by Wagnières et al. [27] and later referred to as the standard method, was used to measure the phantoms. Its principle consists of measuring the diffuse reflectivity $R_{\mathrm{d}}$ and the effective attenuation coefficient $\mu_{\text {eff. }} \mu_{\mathrm{a}}$ and $\mu_{\mathrm{s}}{ }^{\prime}$ are obtained graphically from a table generated by Monte Carlo simulations relating the measured quantities to the optical coefficients. The effective attenuation coefficient was obtained by fitting the exponential decay of the fluence rate versus the sample depth. The fluence rate was measured with an isotropic detector designed by Bays et al. [28]. The diffuse reflectivity was determined by integration sphere measurements.

After the measurements on the phantoms, the in vivo optical coefficients of the skin of six Caucasian human subjects (three males, three females) were determined at 400,500,633 and $700 \mathrm{~nm}$. The skin was measured on the inner side of the left forearm. One measurement at each wavelength was performed per subject.

\section{RESULTS}

\section{Optical Phantoms}

Figure 4 presents the predicted values (dashed and dotted lines) and the optical coefficients of the liquid phantoms measured with the SFDR 


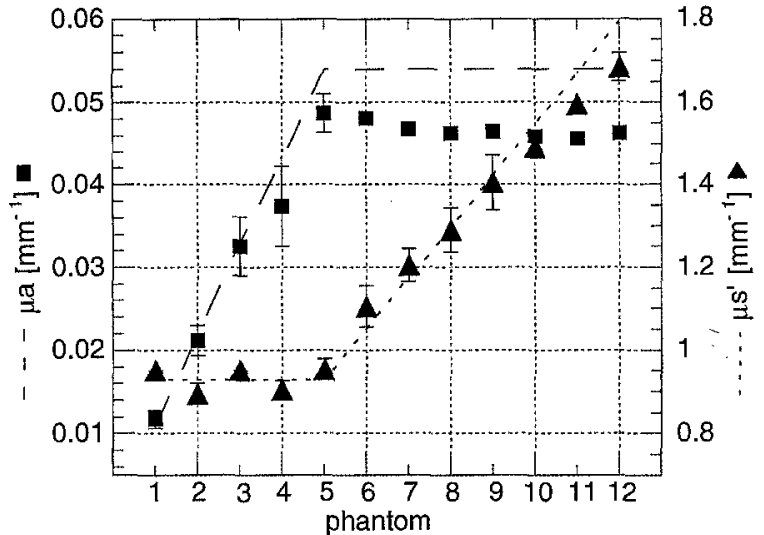

Fig. 4. Results obtained with the SFDR method on 12 different optical phantoms (symbols) in comparison with the predicted values (lines).

method (symbols) at a wavelength of $633 \mathrm{~nm}$. Four measurements were performed on each phantom and the error bars correspond to the standard deviation on $\mu_{\mathrm{a}}$ and $\mu_{\mathrm{s}}{ }^{\prime}$. The concentration of ink in the phantoms was increased linearly from phantom 1 to 5 , resulting in a linear increase of the measured values of $\mu_{\mathrm{a}}$, while the measured $\mu_{\mathrm{s}}{ }^{\prime}$ remained constant. From phantom 5 to 12 , the concentration of Lipovenös in the phantoms was increased linearly, resulting in a linear increase of the measured values of $\mu_{\mathrm{s}}^{\prime}$, while $\mu_{\mathrm{a}}$ decreased about $8 \%$. Phantoms 2 and 4 were also measured with the standard method. Absorption coefficients of $\mu_{\mathrm{a}}=0.016 \mathrm{~mm}^{-1}, 0.032 \mathrm{~mm}^{-1}$ and reduced scattering coefficients of $\mu_{\mathrm{s}}^{\prime}=0.91 \mathrm{~mm}^{-1}$ and $0.86 \mathrm{~mm}^{-1}$ were obtained.

\section{Tissue Samples}

The in vivo optical coefficients of skin of six Caucasian human subjects (three males, three females) were determined with the SFDR method at 400, 500, 633 and $700 \mathrm{~nm}$. The values are presented in Fig. 5 and Table 2. The errors given in the table correspond to the standard deviation as obtained on the six subjects. As expected, the absorption and reduced scattering coefficients of human skin decrease with increasing wavelength.

\section{DISCUSSION}

\section{SFDR Measurements on Optical Phantoms}

The optical coefficients were determined with the non-invasive SFDR method based on the illumination of the investigated sample with spatially periodically modulated light. The absorption coefficients of the phantoms measured with the SFDR correspond well to the predicted ones. The maximum deviation between the predicted and measured values of $\mu_{2}$ is $19 \%$. The values determined with the SFDR method are generally lower than the predicted ones. This can be due to an overestimation of the absorption coefficient from the absorption measurement with the spectrophotometer. The ink solution was assumed to absorb only, therefore any scattering of the ink particles was disregarded. However, depending on the ink particle size, the absorption coefficient could be $30 \%$ lower than the one deduced from the absorption measurement with a spectrophotometer, as investigated for India ink by Madsen et al. [29]. The decrease of the absorption coefficient of $8 \%$ from phantom 5 to 12 while increasing the scattering coefficient by adding Lipovenös is probably due to an interaction between, the absorbing ink particles and the Lipovenös. Phantoms 2 and 4 were also measured with the standard method. The absorption coefficients $\mu_{\mathrm{a}}=0.016 \mathrm{~mm}^{-1}$ and $0.032 \mathrm{~mm}^{-1}$ were obtained for phantoms 2 and 4 , respectively. These values are lower than those predicted by $25 \%$ and $27 \%$, respectively. Comparing $\mu_{\mathrm{a}}$ obtained with the SFDR and the standard method and the predicted values, resulting in less than $27 \%$ deviation between them, it can be concluded that the SFDR method is a reliable method to determine the absorption coefficient in the range of values corresponding to the typical absorption of red light by soft tissues with an error of $20 \%$.

The deviation between the reduced scattering coefficient predicted and measured with the SFDR method is always lower than $9 \%$, i.e. essentially within the error bars. The scattering coefficients $\mu_{\mathrm{s}}{ }^{\prime}=0.91 \mathrm{~mm}^{-1}$ and $0.86 \mathrm{~mm}^{-1}$ were obtained for phantoms 2 and 4 with the standard method. These values are lower than those predicted by $2 \%$ and $7 \%$, respectively, whereas the values obtained with the SFDR are lower by $2 \%$ and $5 \%$, respectively. After comparing the results obtained with the SFDR, standard method and the predicted values of $\mu_{\mathrm{s}}{ }^{\prime}$, resulting in less than $9 \%$ deviation between them, it can be concluded that the SFDR method is a reliable method to determine the reduced scattering coefficient in the range of values corresponding to the typical scattering of soft tissue. 


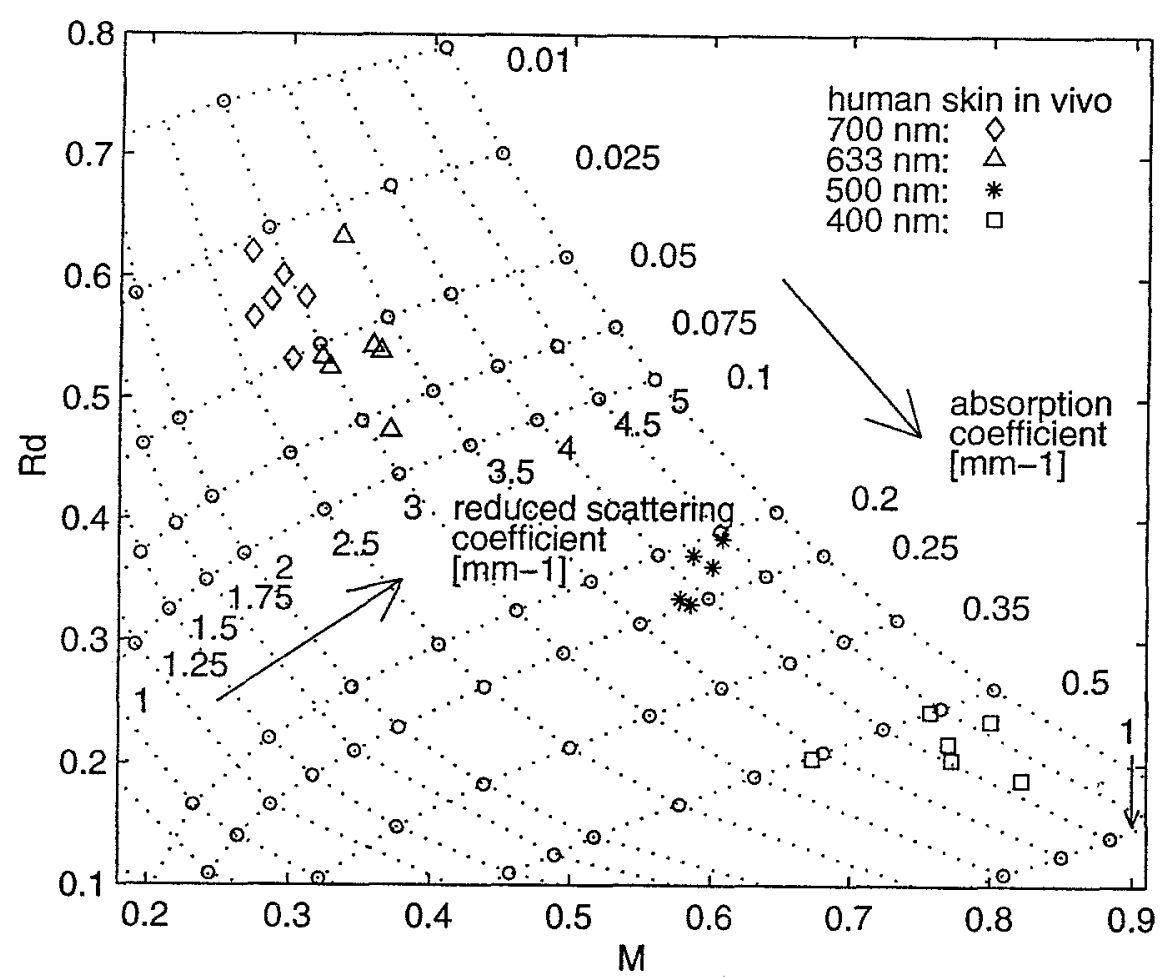

Fig. 5. Results of in vivo skin measurements, obtained with the SFDR method from the Monte Carlo-generated table.

Table 2. Optical coefficients of skin measured with the SFDR method

\begin{tabular}{lll}
\hline & $\mu_{\mathrm{a}}\left(\mathrm{mm}^{-1}\right)$ & $\mu_{\mathrm{s}}{ }^{\prime}\left(\mathrm{mm}^{-1}\right)$ \\
\hline $400 \mathrm{~nm}^{\mathrm{a}}$ & $0.6 \pm 0.1$ & $4.15 \pm 0.44$ \\
$500 \mathrm{~nm}$ & $0.23 \pm 0.02$ & $4.09 \pm 0.28$ \\
$633 \mathrm{~nm}$ & $0.062 \pm 0.013$ & $3.20 \pm 0.20$ \\
$700 \mathrm{~nm}$ & $0.038 \pm 0.008$ & $2.87 \pm 0.15$ \\
\hline
\end{tabular}

${ }^{\text {a }}$ Increased error due to bandwidth of $\pm 20 \mathrm{~nm}$.

The relative errors on the optical coefficients can be calculated with equations (3a) and (3b), with the sensitivities of the method as presented in Fig. 2(a-d) and with the estimated relative measurement errors of $M_{\mathrm{m}}$ and $R_{\mathrm{d}, \mathrm{m}}$ as is shown in Table 1. Essentially, three sources of error occur with the SFDR method.

1. Specular reflections can be detected in certain situations by the CCD camera. This has been observed with phantoms which remained at ambient temperature for several hours. The oil in the Lipovenös formed little drops speculary reflecting light to the CCD camera.

2. An incorrect determination of the centre point of the backscattered pattern also generates artefacts as it leads to an underestimation of the modulation.
3. For all the measurements performed on both the investigated sample and the $100 \%$ reflecting reference, a lambertian light distribution is assumed and moreover, it is assumed that the diffuse reflectivity of the sample (tissue or reference) detected by the CCD camera under a small solid angle is proportional to the diffuse reflectivity coincident in a solid angle of $2 \pi$. To assess this, two simulations were carried out with typical values of the optical coefficients $\left(\mu_{\mathrm{a}}=0.05 \mathrm{~mm}^{-1}, \quad \mu_{\mathrm{s}}{ }^{\prime}=3 \mathrm{~mm}^{-1}, f_{\mathrm{p}}=0.5 \mathrm{~mm}\right)$. The first was used to calculate the modulation consisting all photons backscattered by the tissue, whereas the second only consisted of detecting the photons leaving the tissue under a small angle of $1.25^{\circ}$. Comparing both modulations resulted in negligible differences smaller than $0.4 \%$. If the distribution of the light backscattered from the samples is not lambertian this could lead to an error in the determination of the optical properties. An adaptation of the Monte Carlo simulation to the detection geometry could avoid this source of error.

\section{Tissue}

The relative errors corresponding to the standard deviations of the optical coefficients of 
skin measured on different subjects range between $8 \%$ and $21 \%$ for $\mu_{\mathrm{a}}$ and $5 \%$ and $11 \%$ for $\mu_{\mathrm{s}}{ }^{\prime}$, depending on the wavelength. Due to the large illumination area, the fluctuations generated by tissue inhomogeneities are small (Table 2). The in vivo optical coefficients of human skin have been measured by Kienle et al. [25] and Graaff et al. [15] at $633 \mathrm{~nm}$ and $660 \mathrm{~nm}$, respectively. Kienle et al. based their measurements on a three-layer model and Graaff et al. on a semi-infinite model. They determined the coefficients using spatially resolved reflectance methods (SRR). The values obtained by Kienle et al. were: $\mu_{\mathrm{a}}=$ $0.067 \mathrm{~mm}^{-1}, \mu_{\mathrm{s}}{ }^{\prime}=1.62 \mathrm{~mm}^{-1} ; \mu_{\mathrm{a}}=0.0026 \mathrm{~mm}^{-1}$, $\mu_{\mathrm{s}}{ }^{\prime}=1.2 \mathrm{~mm}^{-1}$ and $\mu_{\mathrm{a}}=0.096 \mathrm{~mm}^{-1}, \quad \mu_{\mathrm{s}}{ }^{\prime}=$ $0.53 \mathrm{~mm}^{-1}$ for the superficial ( $1 \mathrm{~mm}$ thick), the second ( $1 \mathrm{~mm}$ thick) and the third layers (semiinfinite), respectively. Graaff et al. obtained $\mu_{\mathrm{a}} \approx 0.01 \mathrm{~mm}^{-1}$ and $\mu_{\mathrm{s}}{ }^{\prime} \approx 1.1 \mathrm{~mm}^{-1}$. The value of $\mu_{a}$, determined with the SFDR method is between the values reported by Graaff and Kienle, whereas the reduced scattering coefficient is twice as large (see Table 2). This difference can be due to the different nature of the measuring techniques. Indeed, the SRR method is based on illumination at a single point and detection of the diffuse reflectance at a distance of several millimetres from the illumination spot. Photons detected far (several $\mathrm{mm}$ ) from the illumination point have propagated deeply in the medium and are therefore probing deep-seated tissue. Such photons play a less important role in the SFDR method as most of the detected signal is due to the backscattered photons near the illumination. This means that different tissue volumes are investigated with these methods or in other words, the SFDR method gives values which correspond to more superficial tissues than the values given by the SRR method.

Another effect may also explain the discrepancy between the values of $\mu_{\mathrm{s}}{ }^{\prime}$ measured with the SFDR method and the values reported in the literature. Due to the roughness of the tissue-air interface, the light is not reflected speculary in only one direction, and therefore detected in part by the camera despite of the angle between the illumination and detection axis being $7^{\circ}$. This results in an overestimation of both $R_{\mathrm{d}, \mathrm{m}}$ and $M_{\mathrm{m}}$, and overestimated values of $\mu_{\mathrm{s}}^{\prime}$ (Fig. 5). As a precise description of the roughness of skin is extremely difficult to perform, an improvement of the SFDR method consists of avoiding the detection of specular reflections. This can be done by investigating
Table 3. Influence of the anisotropy factor $g$ on the diffuse reflectivity and the modulation

\begin{tabular}{llll}
\hline & $g=0$ & $g=0.8$ & $g=0.95$ \\
\hline$\mu_{\mathrm{a}}=0.05 \mathrm{~mm}^{-1}$ & $R_{\mathrm{d}}=0.277$ & $R_{\mathrm{d}}=0.262$ & $R_{\mathrm{d}}=0.257$ \\
$\mu_{\mathrm{s}}{ }^{\prime}=0.5 \mathrm{~mm}^{-1}$ & $M=0.226$ & $M=0.114$ & $M=0.102$ \\
$\mu_{\mathrm{a}}=0.05 \mathrm{~mm}^{-1}$ & $R_{\mathrm{d}}=0.616$ & $R_{\mathrm{d}}=0.616$ & $R_{\mathrm{d}}=0.617$ \\
$\mu_{\mathrm{s}}{ }^{\prime}=5 \mathrm{~mm}^{-1}$ & $M=0.563$ & $M=0.502$ & $M=0.495$ \\
\hline
\end{tabular}

the sample in refractive index matching conditions or by the use of polarised illumination light and detecting the backscattered light through a polariser orientated at $90^{\circ}$. The Monte Carlo simulation to generate a conversion table as presented in Fig. 5 has to be adapted to take into account the modifications proposed here. It should be noted that the roughness of the air-tissue interface also plays a role in most other techniques presented in the literature, but its effect on the measured values has never to our knowledge "been corrected.

Detecting of the backscattered light near the illumination point is more sensitive to variations of the anisotropy factor due to the small number of scattering interactions. Monte Carlo simulations were performed for three values of the anisotropy factor $g=0, g=0.8$ and $g=0.95$ and for two sets of $\mu_{\mathrm{a}}$ and $\mu_{\mathrm{s}}{ }^{\prime}$ to evaluate the influence of $g$. The simulation parameters were: $n_{\text {tissue }}=1.37$, semi-infinite geometry, $10^{7}$ photons, $f_{\mathrm{p}}=0.5 \mathrm{~mm}^{-1}$. The values of $\mu_{\mathrm{a}}$ and $\mu_{\mathrm{s}}{ }^{\prime}$ of these simulations correspond to the range of the skin optical properties at $633 \mathrm{~nm}$ and to the optical properties of the liquid phantoms presented in this study. As is shown in Table 3 , decreasing $g$ increases the modulation, whereas $R_{\mathrm{d}}$ is essentially constant. Decreasing $g$ from 0.95 to 0.8 results, for $\mu_{\mathrm{s}}{ }^{\prime}=0.5 \mathrm{~mm}^{-1}$, in relative errors of $23 \%$ and $19 \%$ for $\mu_{\mathrm{a}}$ and $\mu_{\mathrm{s}}^{\prime}$, respectively and, for $\mu_{\mathrm{s}}{ }^{\prime}=5 \mathrm{~mm}^{-1}$, in relative errors of $\mu_{\mathrm{a}}$ and $\mu_{\mathrm{s}}{ }^{\prime}$ of $4 \%$ and $2 \%$, respectively. The influence of $g$ on the optical properties was determined graphically from the conversion table presented in Fig. 5. As it is known that the anisotropy factor of soft mammalian tissues ranges typically between 0.8 and 0.95 [12], the influence of a variation of $g$ within these limits can be disregarded for the measurements performed with the SFDR method. To determine the optical properties of tissues with a low scattering coefficient $\left(\mu_{\mathrm{s}}^{\prime}<0.5 \mathrm{~mm}^{-1}\right)$, it is 
necessary to investigate the influence of $g$ on the sensitivity and on the measurement error in dependence of the pattern frequency.

Finally, an improvement on the SFDR method is to adapt it to a tissue model with a multilayered structure by considering the amplitudes of the Fourier transformed at multiples of the spatial frequency $f_{\mathrm{p}}$ in order to extract the optical coefficients of the different layers.

\section{CONCLUSIONS}

It has been shown that the SFDR method enables a non-invasive and in vivo determination of the absorption and reduced. scattering coefficients of tissue. It is not even necessary to touch the tissue. The new method was validated with phantoms with known optical properties, moreover, the absorption and reduced scattering coefficients of skin were determined at 400,500,633 and $700 \mathrm{~nm}$. The coefficients obtained at 633 and $700 \mathrm{~nm}$ correspond well to values represented in the literature. A major advantage of the SFDR method is that it uses a relatively large tissue area to determine a mean value of $\mu_{\mathrm{a}}$ and $\mu_{\mathrm{s}}{ }^{\prime}$. Therefore, local tissue inhomogeneities have a low influence on the measurement, resulting in low standard deviations. Finally, it should be noted that the SFDR technique is well-suited to performing endoscopic measurements in most of the hollow organs using the imaging capabilities of endoscopes.

\section{ACKNOWLEDGEMENTS}

The authors thank Dr A. Kienle and Dr R. Bays for useful discussions and Prof $H$. van den Bergh for his enthusiastic support of this work. They are grateful to the Swiss Fonds National, the Swiss National Priority Program in Optics, and the fund CHUV-EPFL-UNIL for financial support.

\section{REFERENCES}

1. Ishimaru A. Wave propagation and scattering in random media. New York: Academic Press, 1978

2. Patterson MS, Wilson BC, Wyman DR. The propagation of optical radiation in tissue: 1 . Models of radiation transport and their application. Lasers Med Sci 1991; 6:155-68

3. Wilson BC, Patterson MS. The physics of photodynamic therapy. Phys Med Biol 1986; 31:327-60

4. Wilson BC, Adam G. A Monte Carlo model for the absorption and flux distributions of light in tissue. Phys Med Biol 1983; 10:824-30
5. Arridge SR, Cope M, Delpy DT. The theoretical basis for the determination of optical pathlengths in tissue: temporal and frequency analysis. Phys Med Biol 1992; 37:1531-60

6. Star WM. Light dosimetry in vivo. Phys Med Biol 1997; 42:763-87

7. Richards-Kortum R, Sevick-Muraca E. Quantitative optical spectroscopy for tissue diagnosis. Annu Rev Phys Chem 1996; 47:555-606

8. Andersson-Engels S, af Klinteberg C, Svanberg K, Svanberg S. In vivo fluorescence imaging for tissue diagnostics. Phys Med Biol 1997; 47:815-24

9. Henyey LG, Greenstein JL. Diffuse radiation in the galaxy. Astrophys J 1941; 93:70-83

10. v.d.Hulst HC. Light Scattering by Small Particles, New York: Dover, 1981

11. Graaff R, Aarnoudse JG, de Mul FF, Jentink HW. Light propagation parameters for anisotropically scattering media based on a rigorous solution of the transport equation. Appl Opt 1989; 28:2273-9

12. Cheong W, Prahl SA, Welch AJ. A review of the optical properties of biological tissues. IEEE $J$ Quantum Electron 1990; 26:2166-85

13. Graaff R, Aarnoudse JG, de Mul FF, Jentink HW. Similarity relations for anisotropic scattering in absorbing media. Opt Eng 1993; 32:244-52

14. Yoon G, Prahl SA, Welch AJ. Accuracies of the diffusion approximation and its similarity relations for laser irradiated biological media. Appl Opt 1989; $28: 2250-5$

15. Graaff R, Dassel ACM, Koelink $\mathrm{MH}$ et al. Optical properties of human dermis in vitro and in vivo. Appl Opt 1993; 32:435-47

16. Prahl SA, Vitkin IA. Determination of optical properties of turbid media using pulsed photothermal radiometry. Phys Med Biol 1992; 37:1203-17

17. Patterson MS, Chance B, Wilson BC. Time resolved reflectance and transmittance for noninvasive measurement of tissue optical properties. Appl Opt $1989 ; 28: 2331-6$

18. Patterson MS, Moulton JD, Wilson BC et al. Frequency-domain reflectance for the determination of scattering and absorption properties of tissue. Appl Opt 1991; 30:4474-6

19. Groenhuis RAJ, Ferwerdea HA, Ten Bosch JJ. Scattering and absorption of turbid materials determined from reflection measurements. 1: Theory. Appl Opt 1983; $22: 2456-62$

20. Groenhuis RAJ, Ten Bosch JJ, Ferwerdea HA. Scattering and absorption of turbid materials determined from reflection measurements. 2: Measuring method and calibration. Appl Opt 1983; 22:2463-7

21. Kienle A, Lilge L, Patterson MS et al. Spatially resolved absolute diffuse reflectance measurements for noninvasive determination of the optical scattering and absorption coefficients of biological tissue. Appl Opt 1996; 35: 2304-14

22. Bays $\mathrm{B}$, Wagnières $\mathrm{G}$, Robert $\mathrm{D}$ et al. Clinical determination of tissue optical properties by endoscopic spatially resolved reflectometry. Appl Opt 1996; 35:1756-66

23. Wang L, Jacques SL. Monte Carlo Modeling of Light Transport in Multi-Layered Tissues in Standard C, University of Texas MD, Anderson Cancer Center, Houston, Texas, 1992

24. Wagnières, G. Photochimiotherapie et photodetection du cancer a l'aide de photosensibilisateurs ou de 
colorants fluorescents, These No. 1024, EPFL, Lausanne, Switzerland, 1992

25. Kienle A, Lilge L, Patterson MS. Investigation of multi-layered tissue with in vivo reflectance measurements. Proc SPIE 2326, Lille, 1994:212-21

26. van Staveren HJ, Moes CJM, van Marle J et al. Light scattering in intralipid $10 \%$ in the wavelength range of 400-1000 nm. Appl Opt 1991; 30:4507-14

27. Wagnières G, Cheng S, Zellwegger M et al. An optical phantom with tissue-like properties in the visible for use in PDT and fluorescence spectroscopy. Phys Med Biol 1997; 42:1-12

28. Bays B, Wagnières $G$, Robert D et al. A threedimensional optical phantom and its applications in photodynamic therapy. Lasers Surg Med 1997; 21:227-34

29. Madsen SJ, Patterson MS, Wilson BC. The use of India ink as an optical absorber in tissue-simulating phantoms. Phys Med Biol 1992; 37:985-93

30. van Staveren HJ, Beek JF, Ramaekers JWH et al. Integrating sphere effect in whole bladder wall photodynamic therapy: I. $532 \mathrm{~nm}$ versus $630 \mathrm{~nm}$ optical irradiation. Phys Med Biol 1994; 39:947-59

31. Kienle A, Patterson MS. Improved solutions of the steady-state and time-resolved diffusion equations for reflectance from a semi-infinite turbid medium. J Opt Soc A 1997; 14:246-54

Paper received 28 July 1997; accepted after revision 3 February 1998. 